\title{
Elektronenoptische Untersuchungen an Tellur
}

\author{
P. KLein und K. Kleinhenz
}

II. Physikalisches Institut der Universität Köln

(Z. Naturforsch. 23 a, 530-532 [1968] ; eingegangen am 5. Januar 1968)

\begin{abstract}
Starting from bulk single crystals, tellurium has been prepared for transmission electron microscopy. The foil orientation was $\{10 \overline{1} 0\}$, two planes of the reciprocal lattice being projected into the electron diffraction image plane. Dislocations and small angle grain boundaries were observed and could be explained by considering glide on $\{10 \overline{1} 0\}$ and $(0001)$. Hints for a "rhombohedral" view of the tellurium lattice were found.
\end{abstract}

Die Eigenschaften von Tellur, seine Weichheit und große Spaltbarkeit, der niedrige Schmelzpunkt, die Bindungsanisotropie und die hohe Ordnungszahl, lassen von vornherein Schwierigkeiten für seine elektronenmikroskopische Untersuchung erwarten. Das ist wohl der Grund, daß sich unseres Wissens bisher erst eine Arbeit mit diesem Thema befaßt. WeIss und HörL ${ }^{1}$ fanden in Tellur-Aufdampfschichten bei günstiger Orientierung der zusammenwachsenden Kristalle Korngrenzen, die sich als symmetrische tilt-boundaries deuten ließen. Jedoch ist man dabei von der zufälligen Keimorientierung abhängig. Informationen über die im makroskopischen Kristall vorliegenden Baufehler kann man nur erhalten, wenn man vom massiven Kristall ausgeht. Das ist in der vorliegenden Arbeit geschehen. Es werden Verfahren zur Herstellung durchstrahlbarer Proben beschrieben und versucht, die beobachteten Baufehler mit kristallographischen Modellvorstellungen zu verknüpfen.

\section{Präparation}

Für die Untersuchung standen nach dem CzochralskiVerfahren gezogene Einkristalle zur Verfügung. Die üblichen Verfahren zur Präparation durchstrahlbarer Folien, wie Ätzen, elektrolytisches Polieren oder Kathodenzerstäuben, führten nicht zum Erfolg. Wir führen das zum Teil auf die Anisotropie der Bindungskräfte zurück, zum Teil auf die Zerbrechlichkeit des Materials, so daß durchstrahlbar dünne Probenteile beim Hantieren zerbröckelten. Zwei Verfahren haben sich bei Tellur bewährt:

1. Beim elektrolytischen Ätzen in ca. 10-normaler $\mathrm{KOH}$ bei einer Spannung von etwa $2 \mathrm{~V}$ wird der Kristall unregelmäßig blättrig abgetragen. Die Flitter kann man abschwemmen und mit Trägernetzen einsammeln.

2. Bei Quecksilber-dotiertem Tellur sind die Spaltflächen $\{10 \overline{1} 0\}$ nicht mehr glatt, sondern mit nadeligen

1 J. Weiss u. E. M. Hört, Verhandl. Deutsch. Phys. Ges. 6, 377 [1966].
Flittern bedeckt. Diese Flitter sind zu einem großen Teil durchstrahlbar; ihre Länge (in $c$-Richtung) kann mehrere $\mathrm{mm}$, ihre Breite einige $\mu \mathrm{m}$ betragen. $\mathrm{Ob}$ die veränderten Spalteigenschaften auf orientierte Ausscheidung der Dotierung auf den Spaltflächen zurückzuführen sind, ließ sich durch Durchstrahlungs- und Reflexionsbeugung nicht feststellen.

\section{Ergebnisse}

\section{Orientierung}

Als Folienebene wird man, dem Herstellungsververfahren entsprechend, die Spaltflächen $\{10 \overline{1} 0\}$ erwarten. Bei Einstrahlung senkrecht hierzu ergäbe sich als Elektronenbeugungsdiagramm ein zweizähligrechtwinkliges Punktdiagramm mit einem Verhältnis der Punktabstände in $c$-Richtung und senkrecht dazu von 0,378 (Abb. 1). Die beobachteten Werte (z. B.

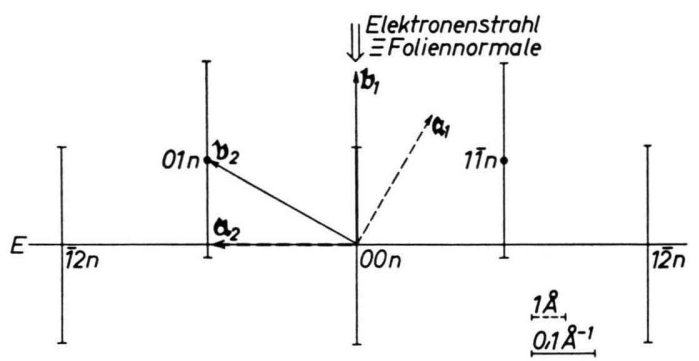

Abb. 1. Orientierung von direktem $\left(\mathfrak{a}_{i}\right)$ und reziprokem $\left(\mathfrak{b}_{i}\right)$ Gitter (r- und $\mathfrak{r}^{*}$-Achse aus Papierebene heraus) sowie Ewald-Schnitt E.

Abb. $2^{*}$ ) liegen um 0,75 (Abweichungen von $\pm 0,02$ sind auf leichte Verkippung der Probe gegen den Elektronenstrahl zurückzuführen). Zur Deutung muß man annehmen, daß durch den „Ewald-Schnitt“ der Ausbreitungskugel nicht nur eine Ebene des rezipro-

* Abb. 2-5 auf S. 530 a, Abb. 7-10 auf S. 530 b. 
P. Klein und K. Kleinheinz, Elektronenoptische Untersuchungen an Tellur (S.530).
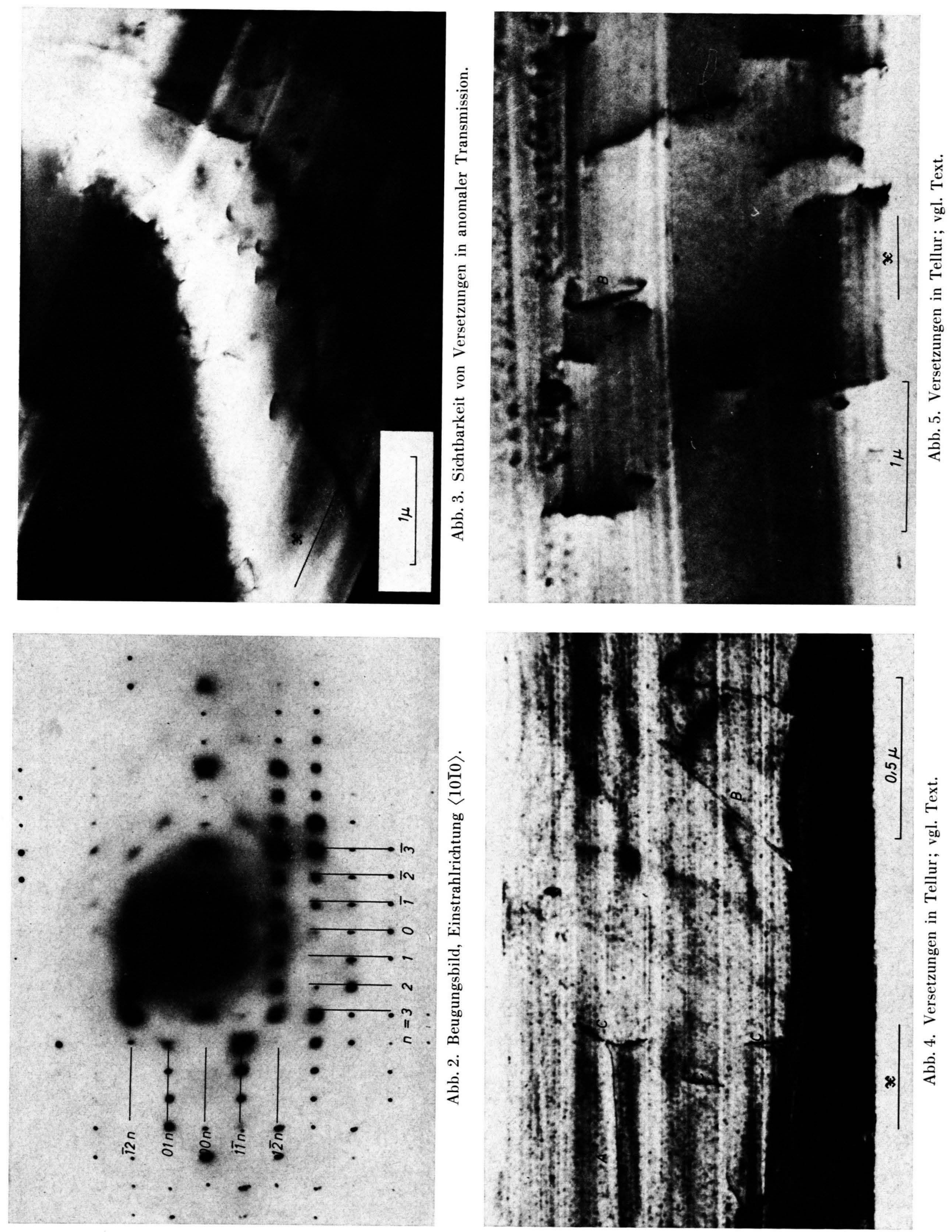

Zeitschrift für Naturforschung 23 a, Seite 530 a. 

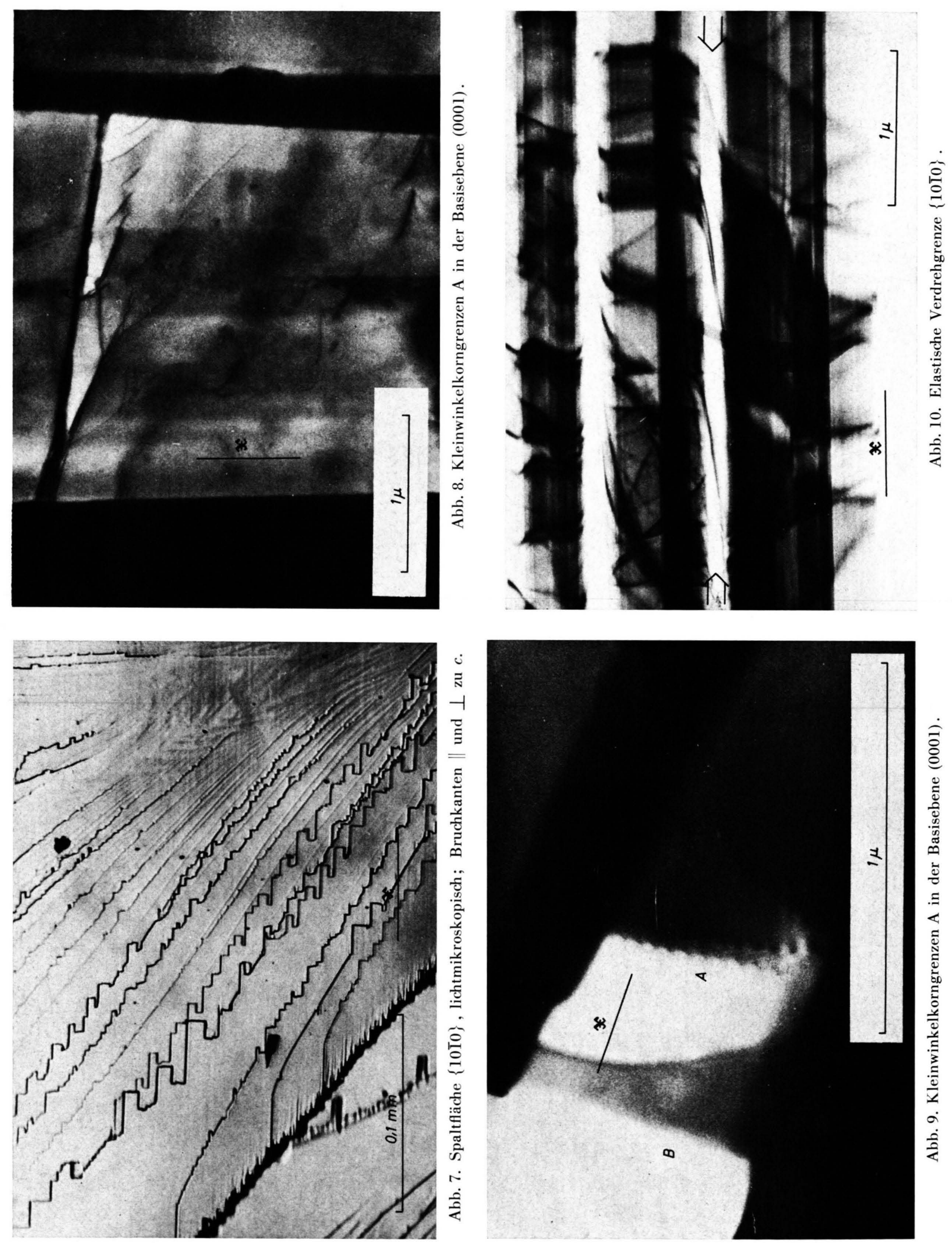

Zeitschrift für Naturforschung 23 a, Seite 530 b. 
ken Gitters abgebildet wird, sondern überdies die benachbarte, wie in Abb. 1 angedeutet. Die seit langem bekannte ${ }^{2}$ Verstärkung der Nebenmaxima der Beugungsstäbchen bei dicken Kristallen ist hier also besonders ausgeprägt. Das Herüberreichen benachbarter reziproker Gitterebenen in die Ewald-Kugel verschwindet erst bei Kristalldicken über 0,4 $\mu \mathrm{m}$. Bemerkenswert ist ferner das Erscheinen von diffusen Strichen, die mit der $c$-Richtung $\approx 45^{\circ}$ einschließen. Es ist möglich, daß diese von thermischen Schwingungen in den "rhomboedrischen“ Kristallrichtungen von Tellur (s. u.) herrühren.

\section{Versetzungen}

Für die Beobachtung im Abbildungsstrahlengang bedient man sich mit Vorteil der anomalen Transmission ${ }^{3}$. Mit kleinen Strahlströmen (geringe Erhitzung!) lassen sich dann noch Kristalle von 0,5 $\mu \mathrm{m}$ Dicke untersuchen. Als Paar inverser Reflexe, bei denen anomale Transmission besonders ausgeprägt ist, finden sich bei Tellur (0003) und $(000 \overline{3})$. Durch Kippen kann man die Streifen anomaler Transmission über den Kristall bewegen und diesen durchmustern. Häufig findet sich jedoch eine Verankerung der Streifen in Gebieten starker Spannung (Abb. 3).

Beispiele für Versetzungen in breiten Transmissionsgebieten (wenig verbogene Proben) zeigen die Abb. 4 und 5. Bei der Deutung mußten wir uns vorerst auf Identifikation mit plausiblen Versetzungstypen beschränken. Die Bestimmung des BurgersVektors mit dem Beugungskontrastverfahren war bei Tellur nicht möglich, weil durch die Abscheidung der Kohle-Kontamination die Proben während der Messung so stark verformt werden, daß eine genauere Analyse unmöglich ist. Der Bau einer Kombination von Kippung und Objektraumkühlung ist geplant.

Mögliche Typen von Versetzungen erhält man vor allem aus dem Studium des Gleitverhaltens. Die Gleitung bei Zugversuchen wurde von SToKes et al. ${ }^{4}$ untersucht; sie verläuft senkrecht zur $c$-Richtung auf Prismenflächen $\{10 \overline{1} 0\}$. Für den Mechanismus der Gleitung werden Stufenversetzungen parallel (Abb. 6, a) und Schraubenversetzungen senkrecht $c$ (Abb. 6 , b) mit dem Burgers-Vektor $\frac{1}{3} a\langle 11 \overline{2} 0\rangle$ vorgeschlagen. Die erstgenannte Versetzung, die, wie man sieht, in der Folienebene verläuft, findet sich im Elektro-

2 F. Kirchner, Ann. Phys. 13, 38 [1932].

3 H. Hashimoto, A. Howie u. M. J. Whelan, Phil. Mag. 5, 967 [1960].

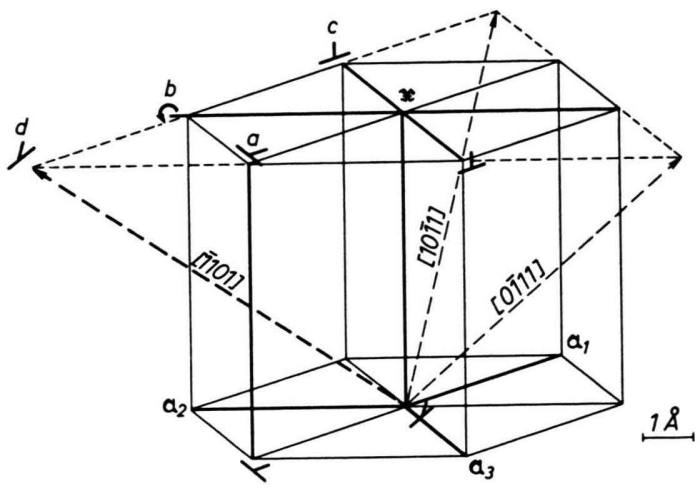

Abb. 6. Orientierung von Versetzungen und Lage der rhomboedrischen Achsen (vgl. Text).

nenmikroskop als wenig geschwungener langgezogener Versetzungsschatten (z. B. Abb, 4, A) .

Makroskopisch, z. B. bei Zugversuchen, sind keine weiteren Gleitmöglichkeiten vorhanden, da die erforderlichen Schubspannungen nicht vorkommen können ${ }^{4}$. Mikroskopisch kann man jedoch das Auftreten örtlicher Spannungen erwarten, die diese Schubspannungen liefern. Wichtig ist dabei, daß dann auch Gleitung auf der Basisebene (0001) möglich ist. Dafür gibt es eine Reihe von Hinweisen. So findet man z. B. auf Spaltflächen von Tellur Stufen, deren generelle Richtung von der örtlichen Spannung abhängt, die sich jedoch aus kurzen Stücken parallel und solchen senkrecht zu $c$ zusammensetzen (Abb. 7). Auch im Elektronenmikroskop findet man Bruchkanten von Kristallnadeln, die fast immer in der Basisebene verlaufen (z. B. Abb. 9, B). Die Gleitung auf (0001) erhält man vermittels Stufenversetzung der Richtung $\langle 11 \overline{2} 0\rangle$ mit dem Burgers-Vektor $\langle 10 \overline{\mathrm{I}} 0\rangle$ („eingeschobene“ Ebene: zweitdichtestbesetzte Netzebene $\{10 \mathrm{~T} 0\}$ ) (vgl. Abb. 6, c) oder solchen der Richtung $\langle 10 \mathrm{~T} 0\rangle$ mit dem Burgers-Vektor $\frac{1}{3} a\langle 11 \overline{2} 0\rangle$ ( „eingeschobene“ Ebene: zweitdichtestbesetzte Netzebene $\{11 \overline{2} 0\})$. Erstere sind $60^{\circ}$ (A), letztere $30^{\circ}$ (B in Abb. 5) zur Folienebene geneigt; das Verhältnis der Längen ihrer Projektionen in die Bildebene ist $\approx \frac{1}{3}$.

Die Gleitung auf (0001) zeigt, daß die „homöopolare“ Bindung in Kettenrichtung die „van der Waals"-Bindung zwischen diesen keineswegs so wesentlich überwiegt, wie häufig dargestellt. Diese Aussage findet sich bereits in Rechnungen von STRANSKI

4 R. I. Stokes, T. L. Jonston u. C. H. Li, Acta Met. 9, 415 [1961]. 
et al. ${ }^{5}$; man kann sie auch direkt aus der Kristallstruktur von Tellur entnehmen. Das Gitter besteht nämlich, wie man sich mit Hilfe eines Gittermodells leicht überzeugt, aus nur wenig verzerrten kubischprimitiven Elementarzellen, wobei das Verhältnis der Abstände zum nächsten Kettennachbarn bzw. zum Nachbarn der nächsten Kette wie $0,83: 1$ ist. Faßt man 27 dieser Zellen zu einer zusammen, so erhält man ein rhomboedrisches Gitter mit den aufgespannten Gittervektoren [T101], [0T11], [10T1] (Abb. 6), die untereinander $81^{\circ}$ bilden. Projiziert man eine dieser Richtungen in die Folienebene $\{10 \mathrm{~T} 0\}$, so schließt sie mit der $c$-Richtung $48^{\circ}$ ein; dieser Winkel findet sich häufig bei Versetzungen in Tellur, z. B. Abb. 4 B (vgl. Abb. 6,d). Die angegebenen Vektoren liegen in $\{11 \overline{2} 0\}$-Flächen, sind also, senkrecht $c$, um $30^{\circ}$ gegen die Folie geneigt. Aus dieser Orientierung der Versetzungsrichtung errechnet sich für die Foliendicke der vernünftige Wert von 0,25 $\mu \mathrm{m}$, übereinstimmend mit dem für die Versetzungen $\mathrm{C}$, wenn man für diese eine Neigung von $60^{\circ}$ annimmt. Dies und die oben besprochenen diffusen Striche im Beugungsdiagramm werten wir als Hinweis darauf, daß die rhomboedrische Darstellung von Tellur heuristisch wertvoll ist.

5 I. N. Stranski, R. Kaischew u. F. Krastanow, Z. Krist. 88, 325 [1934].

\section{Korngrenzen}

Dem Gleitverhalten entsprechend, sollte man Kleinwinkelkorngrenzen in $\{10 \overline{1} 0\}$ - und (0001)-Flächen erwarten. Die ersteren scheinen bei der Präparation zerstört zu werden ${ }^{1}$; sie konnten nicht zweifelsfrei beobachtet werden. Dagegen finden sich häufig Korngrenzen in der Basisebene (Abb. 8, A), mit denen meist weitere Versetzungssysteme zusammenhängen, und die man manchmal in einzelne Versetzungen fast senkrecht zur Probenebene auflösen kann (Abb. 9, A). Ebenso häufig ist jedoch der Fall, daß die Orientierungsänderung durch elastische Verbiegung, ohne Versetzungen, vermittelt wird. In Abb. 10 zeigen die in einem schmalen Bereich $\Rightarrow$ abfallenden Extinktionsbänder eine solche elastische Verdrehungsgrenze (twist-boundary) in $\{10 \overline{1} 0\}$; die Dicke der elastisch deformierten Schicht beträgt etwa $0,2 \mu \mathrm{m}$. Auch in $c$-Richtung lassen sich dünne, fehlerfreie Folien sehr stark verbiegen. Es scheint demnach so, als resultiere die ungewöhnliche Spröde von Tellur aus der leichten Erzeugbarkeit ${ }^{4}$ von Oberflächendefekten.

Herrn Prof. Jaumann danken wir für die Anregung $\mathrm{zu}$ dieser Arbeit und sein förderndes Interesse, Herrn Dr. Grosse und Herrn P. Runow für wertvolle Hinweise, der Stiftung Volkswagenwerk für die Bereitstellung des Elektronenmikroskops. 